\title{
VISIBILIDADE CURRICULAR PARA O EXCESSO DE PESO: CONTRIBUIÇÃO DA ANÁLISE TEMÁTICA
}

\author{
Maria Magaly Medeiros ${ }^{1}$, Rosana Vilela $^{1}$ e Andrea Vanderlei Fregadolli ${ }^{1}$ \\ 1Universidade Federal de Alagoas, Brasil, magalymedeiros@uol.com.br; zanavilela@gmail.com; deadoutorado@hotmail.com
}

\begin{abstract}
Resumo. Introdução: O excesso de peso é entendido como um dos maiores problemas de saúde pública no mundo. Objetivo: Este estudo teve por objetivo identificar estratégias educativas para enfrentar a invisibilidade da temática "excesso de peso no currículo de medicina", a partir de estudantes inseridos no internato de uma universidade pública do nordeste brasileiro. Métodos: Optou-se pela metodologia de cunho qualitativo, cujas respostas ao questionamento sobre o tema foram submetidas a uma análise temática, em que se procurou apreender as sugestões para o aprimoramento de seu ensino/aprendizagem. Resultados: Os resultados apontaram duas categorias de sugestões: a primeira envolveu medidas com resultado em médio prazo, como o incentivo ao estilo de vida saudável no ambiente da escola médica; e a segunda tratou de medidas de resultados em curto prazo, como as intervenções educativas visando melhorar as competências dos estudantes na abordagem do excesso de peso. Conclusões: Foi possível concluir que há necessidade de intervenções educativas eficazes durante a graduação com o intuito de melhorar as competências em medicina do estilo de vida e na abordagem do excesso de peso. Assim, os futuros profissionais de saúde estarão mais bem equipados para ajudar os pacientes na adoção e manutenção de comportamentos mais saudáveis.
\end{abstract}

Palavras-chave: Obesidade; Sobrepeso; Currículo; Educação de Graduação em Medicina; Medicina do Estilo de Vida.

\section{CURRICULAR VISIBILITY FOR OVERWEIGHT: CONTRIBUTION OF THEMATIC ANALYSIS}

Abstract. Introduction: Overweight is understood as one of the biggest public health problems in the world. Objective: This study aimed to identify educational strategies to face the invisibility of the issue of overweight in the medical curriculum, from students in boarding schools and a public university in north-eastern Brazil. Method: We opted for the qualitative methodology, where the answers to questions on the theme were subjected to a thematic analysis, in which we sought to apprehend the suggestions for the improvement of their teaching / learning. Results: The results showed two categories of suggestions. The first involved measures with medium-term results, such as encouraging a healthy lifestyle in the medical school environment. The other dealt with shortterm outcome measures, such as educational interventions aimed at improving students' skills in overweight approaching. Conclusions: It is concluded that there is a need for effective educational interventions during graduation aiming to improve skills in lifestyle medicine and in the approach to overweight. Thus, future health professionals will be better equipped to assist patients in adopting and maintaining healthier behaviours.

Keywords: Obesity; Overweight; Curriculum; Undergraduate Medical Education; Lifestyle Medicine.

\section{INTRODUÇÃO}

A Organização Mundial de Saúde (OMS) aponta a obesidade como um dos maiores problemas de saúde pública no mundo. A projeção é que, em 2025, cerca de 2,3 bilhões de adultos estejam com sobrepeso e mais de 700 milhões, obesos. No Brasil, a obesidade vem crescendo cada vez mais. Alguns levantamentos indicam que $50 \%$ da população está acima 
do peso, ou seja, na faixa de sobrepeso e obesidade (Associação Brasileira para o Estudo da Obesidade e da Síndrome Metabólica [ABESO], 2014).

O EP e a obesidade têm caráter múltiplo e heterogêneo, envolvem não apenas fatores biológicos e de causa individual, mas uma integração de fatores históricos, econômicos, sociais e culturais. Não são apenas os aspectos relacionados à dieta ou ao sedentarismo que devem ser avaliados, mas as condições de trabalho, moradia, segurança, rede de abastecimento e globalização, que explicam os fatores proximais que, usualmente, se incluem nos modelos causais das doenças e agravos à saúde (Brasil, 2014).

Os médicos têm um papel especial em ajudar os pacientes a fazer mudanças no estilo de vida e são mais confiáveis e eficazes se forem modelos deste estilo de vida. No entanto, poucas escolas médicas incorporaram a Medicina do estilo de vida em seus currículos (Malatskey, Essa-Hadad, Willis, \& Rudolf, 2019).

Vários estudos demonstram que os médicos, inclusive os da atenção primária, não abordam as medidas preventivas (Colbert \& Jangi, 2013; Block, Desalvo, \& Fisher, 2003), poucos fazem o tratamento de maneira adequada (Leedham-Green, Pound, \& Wylie, 2016) e exibem atitudes preconceituosas que desmotivam o cuidado da pessoa com EP (Fang, Gillespie, Crowe, Popeo, \& Jay, 2019; Pantenburg et al., 2012). As principais barreiras descritas são o pouco conhecimento adquirido no período de formação, sobretudo no que se refere às causas da obesidade, fisiologia, diagnóstico e tratamento. Além disso, o preconceito, algumas vezes não percebido, juntamente com o pouco tempo despendido na consulta, ajudam a piorar o cuidado das pessoas com EP (Leedham-Green et al., 2016).

Os dados epidemiológicos evidenciam a epidemia de EP no mundo, mas os profissionais da saúde ainda não estão preparados para uma atuação adequada. Vários fatores estão envolvidos na "invisibilidade" do EP, tais como: falta de reconhecimento como doença crônica; desconhecimento da fisiopatologia, dos fatores biopsiquicossociais e das opções medicamentosas; falta de tempo para examinar o paciente; poucos medicamentos disponíveis (Yanovski \& Yanovski, 2014) e o preconceito para com as pessoas com EP. O estudo de Vitolins, Crandall, Miller, Ip, Marion e Spangler (2012) demonstrou que a falta de treinamento sobre o assunto durante a graduação também dificulta o cuidado das pessoas com EP ao longo da vida profissional. 
As intervenções educativas terão mais sucesso quando forem projetadas para atender às necessidades dos participantes. A avaliação das necessidades informa o desenho do plano educacional, bem como suas características. Informa, ainda, que tipo de características ou estratégias a intervenção deve adotar para alcançar o resultado desejado (Mogre, Stevens, Aryee, Amalba, \& Scherpbier, 2018; Dacey, Arnstein, Kennedy, Wolf, \& Phillips, 2013).

Ante o exposto e à escassez de estudos nacionais e locais sobre o ensino médico e o EP, buscou-se responder à pergunta: Sob o ponto de vista dos estudantes do internato de medicina, que estratégias educativas dariam maior visibilidade à temática EP durante a graduação? Neste artigo, o objetivo foi identificar estratégias educativas para enfrentar a invisibilidade da temática "excesso de peso no currículo de medicina".

\section{PERCURSO METODOLÓGICO}

Trata-se de um estudo do tipo descritivo, com abordagem qualitativa e delineamento transversal, ou seja, a coleta de dados foi realizada em um único tempo (Selltiz, Wrightsman, \& Cook, 1987).

O estudo é derivado de uma pesquisa mais ampla, intitulada "O manejo clínico do excesso de peso: saberes dos estudantes de medicina". O estudo matricial teve como objetivo geral verificar o conhecimento, a autoconfiança e as atitudes no manejo da pessoa com EP em uma amostra formada por estudantes do internato de medicina de uma universidade pública no nordeste brasileiro. Nesta pesquisa, ao final do questionário, foi acrescida a pergunta aberta: “Quais as sugestões para o aprimoramento do ensino sobre excesso de peso?”. Os dados produzidos por essa pergunta originaram o material a ser apresentado como conteúdo principal deste estudo, com o objetivo de identificar estratégias educativas para enfrentar a invisibilidade da temática "excesso de peso no currículo de medicina".

Para responder à pergunta, foram convidados 32 estudantes que cursavam o estágio de Clínica Médica 2 do internato do curso de medicina de uma universidade pública do nordeste brasileiro. Foram 15 participantes do sexo masculino e 17 do sexo feminino. A pergunta aberta foi respondida por 30 participantes (internos).

O período do internato foi escolhido para realizar o estudo por se tratar da última etapa da formação do médico generalista, portanto, momento adequado para avaliar os 
conhecimentos adquiridos nos ciclos anteriores da graduação. Tratou-se, desse modo, de uma amostra intencional (Yin, 2016).

O questionário, criado no formulário Google Docs, foi enviado por meio eletrônico aos participantes durante o estágio de Clínica Geral, após terem assinado o Termo de Consentimento Livre e Esclarecido (TCLE). O período da coleta das informações foi de junho a novembro de 2018.

Os dados produzidos, a partir da pergunta aberta, foram armazenados, transcritos, sistematizados, categorizados e analisados. A análise de conteúdo foi escolhida e, dentro dessa análise, utilizou-se a proposta de sistema de categorias, com o intuito de organizar e sistematizar os pontos que emergiram das respostas dos participantes. As unidades de registro usadas foram temas, porque, segundo Bardin (2011), a análise temática permite descobrir os sentidos que atravessam a comunicação e a frequência que a compõe indica significados para o objetivo analítico.

A análise temática, de cunho qualitativo, deu especial ênfase à presença dos temas elaborados para responder à pergunta da pesquisa, em detrimento da frequência com que estes aparecem ao longo dos relatos. Sendo assim, o material foi codificado pelas seguintes categorias temáticas: medidas de resultados em médio prazo (incentivo ao estilo de vida saudável no curso de medicina); e medidas de resultados em curto prazo (intervenções educativas visando melhorar as competências dos estudantes na abordagem do EP).

O estudo foi submetido ao Comitê de Ética em Pesquisa da Universidade Federal de Alagoas (UFAL) - Plataforma Brasil - e aprovado com o Parecer $n^{\circ}$ 80644117.4.0000.5013. Com o intuito de preservar o anonimato dos participantes, os seus nomes foram substituídos pela letra $\mathrm{P}$ (participante) seguida de numeração crescente.

\section{RESULTADOS E DISCUSSÃO}

A busca por estratégias é fundamental no processo de desenvolvimento curricular, especialmente no curso que objetiva desenvolver competências médicas de forma integrada e contextual. Ao ser abordado o tema "sugestões para o aprimoramento do ensino sobre EP no âmbito da graduação", durante a pesquisa intitulada "O manejo clínico do excesso de peso: saberes dos estudantes de medicina", emergiram das respostas dos participantes (internos) informações significativas que podem indicar caminhos importantes para a busca 
da excelência do plano pedagógico do curso. Estas informações foram traduzidas em duas categorias: 1) Medidas de resultados em médio prazo: incentivo ao estilo de vida saudável no curso de medicina; 2) Medidas de resultados em curto prazo: intervenções educativas visando melhorar as competências dos estudantes na abordagem do EP.

\subsection{Medidas de resultados em médio prazo: incentivo ao estilo de vida saudável no curso de medicina}

Os internos referem a necessidade de melhorar os hábitos pessoais de saúde dos estudantes de medicina.

\footnotetext{
P4: Fazer, de cada aluno, um paciente, visto que o ganho de peso durante o curso é muito comum, muito em função da nossa péssima qualidade de vida. Em virtude da carga enorme de assuntos pra estudar, carga horária de aulas/estágios, pouco tempo livre e/ou muita correria, grande maioria dos estudantes comem mal (lanches rápidos, por vezes, como sanduíches e pizzas), ficam com pouco tempo pra uma atividade física regular (é possível se adequar com muita disciplina, mas, dentre todas as obrigações que temos, quase sempre a academia é a escolhida pra ser dispensada quando a corda aperta), qualidade do sono prejudicada, tudo que contribui para o ganho de peso e possível obesidade. Logo, se cada aluno, além de ser um estudante (tendo aulas sobre o tema), fosse um paciente, com certeza, seria um tema amplamente aprendido.
}

Os médicos têm um papel especial em ajudar os pacientes a fazer mudanças no estilo de vida, no entanto, poucas escolas médicas incorporaram a Medicina do estilo de vida em seus currículos (Malatskey et al., 2019).

Pesquisas mostraram a associação entre as práticas de saúde dos médicos e a sua capacidade de influenciar o comportamento do estilo de vida de seus pacientes (Oberg \& Frank, 2009; Frank, Dresner, Shani, \& Vinker, 2013). Revelaram, também, que uma prática pessoal mais saudável durante a graduação prediz positivamente as práticas de aconselhamento preventivo dos médicos (Frank, Carrera, Elon, \& Hertzberg, 2007). Malatskey et al. (2019) afirmaram que é improvável que os médicos forneçam uma orientação eficaz se não puderem sustentar comportamentos saudáveis.

\subsection{Medidas de resultados em curto prazo: Intervenções educativas visando melhorar as competências dos estudantes na abordagem do EP}

Dessa categoria emergiram as subcategorias apresentadas a seguir, no Quadro 1. 
Quadro 1. Intervenções educativas visando melhorar as competências dos estudantes na abordagem do EP

\begin{tabular}{|l|}
\hline \multicolumn{1}{|c|}{ Subcategorias } \\
\hline 1- Foco das intervenções educativas \\
\hline 2- Formato metodológico das intervenções educativas \\
\hline 3- Características das intervenções educativas \\
\hline 4- Cenários possíveis para o desenvolvimento das intervenções educativas \\
\hline 5- Avaliação da aprendizagem do estudante \\
\hline
\end{tabular}

\subsubsection{Foco das intervenções educativas}

O foco da maioria das intervenções era melhorar a competências (conhecimento, habilidades e atitudes) em vários tópicos da abordagem do EP, sobretudo a prevenção e o tratamento, com abordagem biopsicossocial.

P25: Tratar sobre as opções terapêuticas, investir na prevenção [...]

P13: Capacitar melhor os acadêmicos para fazer orientação nutricional.

P17: Incluir a temática de forma clara e objetiva, com ênfase no diagnóstico, tratamento farmacológico e não farmacológico [...].

P27: [...] precisa-se tratar os transtornos neuropsiquiátricos que podem levar à obesidade, desde ansiedade a outros sintomas que não se trata antes mesmo da pessoa ser obesa.

Competências fundamentais realçadas nas sugestões foram as referentes ao tratamento farmacológico e não farmacológico da pessoa com excesso de peso. Essas sugestões estão afinadas com os resultados da pesquisa geradora (Medeiros, 2019), que mostraram lacunas sobre os conhecimentos relacionados à conduta medicamentosa e nutricional após o diagnóstico, bem como o momento mais adequado de encaminhar ao especialista.

O Report VIII Contemporary Issues in Medicine: The Prevention and Treatment of Overweight and Obesity da Association of American Medical Colleges discutiu e elaborou o conteúdo para as escolas médicas adotarem, objetivando a implantação do tema na graduação médica (Association of American Medical Colleges [AAMC], 2007). Os conteúdos ressaltados pelos participantes da pesquisa são coerentes com o documento.

\subsubsection{Formato metodológico das intervenções educativas}

Os formatos de ensino e aprendizagem sugeridos pelos participantes incluíram metodologias tradicionais e ativas, como palestras, tutoriais de aprendizagem baseados em problemas, demonstrações, dramatizações, discussões de casos clínicos, aulas práticas. 
P24: Capacitação do estudante por meio de aula/palestra sobre o correto manejo destes pacientes.

P14: Estudos, aulas, focar em casos clínicos e tratamento.

P4: [...] se cada aluno, além de ser um estudante (tendo aulas sobre o tema), fosse um paciente, com certeza, seria um tema amplamente aprendido.

P19: Abordar com mais afinco a temática, sedimentando o conhecimento mediante o acompanhamento de casos ambulatorialmente, o que, em geral, é pouco estimulado.

Uma revisão (Matharu et al., 2014) examinou os métodos de treinamento efetivo para a intervenção em sobrepeso e obesidade na graduação médica. Os estudos utilizaram, com sucesso, uma variedade de métodos de ensino, incluindo treinamento na prática, palestras, dramatização e interação padronizada de pacientes para aumentar a competência dos estudantes de medicina em relação à abordagem do sobrepeso e da obesidade.

Abordagens direcionadas para o conhecimento, habilidades e atitudes devem ser incentivadas. A revisão de literatura desenvolvida por Mogre et al. (2016) mostrou que a maioria das intervenções desenvolvidas é direcionada para melhorar o conhecimento dos profissionais sobre o tema. E essas intervenções proporcionavam pouca mudança no comportamento da prática.

Observou-se ausência de sugestões incorporando educação baseada em tecnologia. A revisão citada anteriormente (Mogre et al., 2016), indica que o uso da tecnologia (computador e internet) leva a ganhos significativos do conhecimento, atitudes positivas, aumento de habilidades de autoavaliação de aconselhamento nutricional e de manejo prático.

\subsubsection{Características das intervenções educativas}

Como característica da abordagem do EP, os participantes sinalizam para a necessidade de atividades transversais, interdisciplinares e interprofissionais, resultando em uma prática colaborativa.

P9: Ela deve ter um espaço de discussão destinado a ela (excesso de peso), portanto, seria interessante incluir esse tema na graduação juntamente com outras patologias crônicas e de grande prevalência. Isso contribuiria para que a obesidade deixasse de ser relacionada apenas a um fator de risco, por sinal, muito determinante para desenvolvimento de outras patologias, e passasse a ser abordada como uma doença, que de fato é. O conhecimento sobre a doença é o principal caminho para melhor abordagem da mesma.

P15: [...] poderia se trabalhar com o tema de obesidade contando com a participação de outros profissionais da área de saúde, não médicos, que ofereceriam uma visão mais ampla e completa do assunto. Este tema deveria ser incluído às aulas de Saúde e Sociedade, além de na Endocrinologia. 
Outro modo de enfrentamento no âmbito pessoal que foi citado pelos entrevistados:

P18: [...] como futuros médicos, acredito que não sejamos capaz de lidar com o processo de sobrepeso sem o trabalho de equipe multiprofissional, porém, como temos pouquíssimo contato com estes profissionais durante a graduação, acabamos não sendo capazes de reconhecer o limite que nossa capacidade atinge e o espaço que podemos direcionar para outros profissionais poderem complementar no cuidado do paciente.

A abordagem do tema EP, como sugerem os internos, deve ter características capazes de desempenhar o cuidado necessário, para tanto, contando com a participação de várias disciplinas médicas, além da Endocrinologia (interdisciplinaridade), bem como outras profissões - Educação Interprofissional (EIP). Nos últimos anos, diversas iniciativas de mudanças na formação das profissões de saúde recomendam a adoção da EIP para avançar em uma nova configuração do trabalho (WHO, 2010; Frenk et al., 2010).

As características das intervenções educativas apontadas pelos participantes são desafiadoras. Elas envolvem a formação docente, dimensão essencial para essa prática pedagógica, requerendo novos saberes e habilidades profissionais que orientem seus estudantes (Batista, Vilela, \& Batista, 2015) até mesmo a aprenderem uns com os outros, como é o caso da educação interprofessional (WHO, 2010; Reeves, Perrier, Goldman, Freeth, \& Zwarenstein, 2013).

\subsubsection{Cenários possíveis para o desenvolvimento das atividades}

Os participantes identificam a atenção primária e secundária como espaços que requerem melhor abordagem sobre o tema EP.

P26: [...] todas as práticas voltadas ao assunto foram realizadas em ambulatório de Endocrinologia, Cardiologia e Saúde da Criança e do Adolescente. Porém, o tema "obesidade", em si, foi pouco trabalhado e ele perpassa todas as áreas da Medicina.

P6: Medidas eficazes na atenção básica contra a obesidade.

P10: Incluir, dentro do bloco de Endocrinologia, esse assunto é reforçar, durante as aulas práticas, a importância da temática para que se transforme em parte de nossa prática rotineira, já que é uma doença que faz parte do nosso dia a dia, mesmo não sendo a queixa principal de muitos desses pacientes.

Os estudantes do internato parecem mais propensos a assimilar a abordagem do indivíduo com EP se eles observarem seus professores e preceptores modelarem o comportamento esperado. A tomada de medidas adequadas sobre cuidados nutricionais, pelos modelos, 
proporcionou modelagem de papéis positivos nos participantes de pesquisa realizada por Scolapio, DiBaise, Schwenk, Macke e Burdette (2008).

Kaplan et al. (2018) demonstraram, por sua vez, que, para melhorar o tratamento da pessoa com EP, são necessários: o diagnóstico formal da doença; a priorização na consulta sobre o tema, assim como o acompanhamento com consultas regulares e a valorização dos programas de cuidado com o peso, além de conhecimento das medicações. A rotação em um programa de cirurgia bariátrica mostrou-se mais promissora quando comparada às rotações em outras disciplinas clínicas (Banasiak \& Murr, 2001). Na pesquisa discutida nesse artigo, observa-se que os participantes têm a compreensão de que, para a aquisição das competências necessárias à abordagem do EP, a temática deve permear todo o curso, não apenas o foco no internato. Infere-se que a prestação de cuidados relacionados ao EP melhora com o aumento do treinamento de habilidades e da quantidade de interações com as pessoas com EP.

P11: O tema deve ser incluído na graduação antes do início do internato, preferencialmente nas clínicas, para que seja abordado de forma detalhada, uma vez que é de grande importância.

Os participantes apontaram a atenção secundária (ambulatório de Endocrinologia, Cardiologia, entre outros) e a atenção primária em saúde como espaços importantes e ricos para o exercício prático sobre EP, especialmente no internato. Estas proposições vão ao encontro das DCN (Brasil, 2014), que apontam a rede básica como um campo potencial e necessário de prática colaborativa no qual vários cursos de formação de profissionais de saúde deverão inserir seus estudantes.

\subsubsection{Avaliação de aprendizagem do estudante}

A avaliação é parte constitutiva das intervenções educativas e propicia o acompanhamento dos avanços e das dificuldades na aprendizagem, por esta razão deve estar relacionada com os objetivos da aprendizagem, devendo, assim, estar voltada para os fins e não somente para os resultados. Nas sugestões dos estudantes do internato, o pleito por mais avaliações sobre o tema surge de forma muito tímida.

Gontijo, Alvim e Castro Lima (2015, p. 211) afirmaram que "nenhum método é capaz de isoladamente avaliar os múltiplos aspectos que envolvem o saber médico. Daí a necessidade de a avaliação combinar diferentes instrumentos e múltiplas observações, com registros sistemáticos". Os estudos de Ockene et al. (2018) e de Fang et al. (2019) 
utilizaram o Exame Clínico Objetivo Estruturado (OSCE) como instrumento de avaliação das habilidades para o manejo da pessoa com EP.

Uma importante ferramenta da avaliação formativa é o feedback. Feedback é a entrega de informações com base na observação direta, visando melhorar o desempenho do estudante. Jug, Jiang, \& Bean (2019) observaram que a literatura sobre educação médica descreve vários métodos para dar feedback com suas facilidades e barreiras. Porém, ainda são poucos os artigos que descrevem a importância de receber feedback. Ressaltam que dar e receber feedback se torna mais fácil com a prática.

\section{CONCLUSÕES}

O reconhecimento da importância ou mesmo a incipiente presença do tema no currículo são insuficientes para promover o necessário manejo clínico da pessoa com excesso de peso.

Os achados desse estudo direcionam para a necessidade do curso de medicina pesquisado investir em medidas de resultados em médio prazo, como incentivo ao estilo de vida saudável no ambiente da escola médica. Bem como, medidas de resultados em curto prazo - Intervenções educativas visando melhorar as competências dos estudantes na abordagem do EP.

Esta conduta é essencial para ampliar as chances de superar a epidemia de doenças relacionadas ao estilo de vida, que está dominando a saúde em todo o mundo. Isso requer a inclusão de um programa de incentivo ao estilo de vida saudável, por meio de intervenções transversais e frequentes no currículo, enfatizando a importância dos comportamentos de saúde pessoal e das habilidades profissionais no apoio à mudança do estilo de vida.

Reitera-se, ainda, a necessidade da construção de intervenções educacionais referentes ao manejo da pessoa com EP, em um contexto interdisciplinar e interprofissional, voltadas para o conjunto de discentes e docentes/preceptores envolvidos na formação profissional.

É importante a criteriosa escolha de métodos e técnicas pedagógicos e de avaliação que, amparados em fundamentos teóricos que expliquem o desenvolvimento das competências necessárias para o manejo de pessoas com EP, possam efetivamente interferir neste processo de adoecimento.

Como ponto forte da pesquisa pode-se citar a integração de sua natureza com o uso da abordagem qualitativa com análise do conteúdo, do tipo temática. Esta aproximação 
permitiu a geração de categorias contendo as sugestões de intervenções educativas, para implementação futura, sobre a abordagem do EP. Outro potencial indicado nessa análise foi mostrar que planejadores e gestores de intervenções educacionais devem ouvir as necessidades e os interesses dos estudantes do curso.

No entanto, o estudo teve, também, limitações. Uma é que a interpretação de natureza subjetiva da pesquisa qualitativa admite que outros pesquisadores possam chegar a diferentes resultados. Outra limitação refere-se ao olhar da pesquisa baseada apenas nos estudantes, embora se tenha encontrado alta consistência com os dados da literatura.

\section{REFERÊNCIAS}

Associação Brasileira para o Estudo da Obesidade e da Síndrome Metabólica. (2014). Mapa da Obesidade. São Paulo.

Association of American Medical Colleges. (2007). Report VIII contemporary Issues in Medicine: the prevention and treatment of overweight and obesity. Washington, USA.

Banasiak, M., \& Murr, M. M. (2001). Medical school curricula do not address obesity as a disease. Obesity Surgery, 11(6), 677-679.

Bardin, L. (2011). Análise de conteúdo. São Paulo: Edições 70.

Batista, N. A., Vilela, R., \& Batista, S. H. (2015). Educação médica no Brasil. São Paulo: Cortez.

Block, J. P., DeSalvo, K. B., \& Fisher, W. P. (2003). Are physicians equipped to address the obesity epidemic? knowledge and attitudes of internal medicine residents. Preventive Medicine, 36(6), 669-675.

Brasil. Ministério da Educação. Conselho Nacional de Educação. (2014). Resolução CNE/CES n. 3, de 20 de junho de 2014. Institui Diretrizes Curriculares Nacionais do Curso de Graduação em Medicina e dá outras providências. Brasília, DF.

Brasil. Ministério da Saúde. Organização Pan-Americana da Saúde. (2014). Perspectivas e desafios no cuidado às pessoas com obesidade no SUS: resultados do Laboratório de Inovação no manejo da obesidade nas Redes de Atenção à Saúde. Brasília: Ministério da Saúde.

Colbert, J. A., \& Jangi, S. (2013). Training physicians to manage obesity--back to the drawing board. The New England Journal of Medicine, 369(15), 1389.

Dacey, M., Arnstein, F., Kennedy, M. A., Wolfe, J., \& Phillips, E. M. (2013). The impact of lifestyle medicine continuing education on provider knowledge, attitudes, and counseling behaviors. Medical Teacher, 35(5), e1149-e1156.

Fang, V., Gillespie, C., Crowe, R., Popeo, D., \& Jay, M. (2019). Associations between medical students' beliefs about obesity and clinical counseling proficiency. BMC Obesity, 6(1), 1-8.

Frank, E., Carrera, J. S., Elon, L., \& Hertzberg, V. S. (2007). Predictors of US medical students' prevention counseling practices. Preventive Medicine, 44(1), 76-81.

Frank, E., Dresner, Y., Shani, M., \& Vinker, S. (2013). The association between physicians' and patients' preventive health practices. Cmaj, 185(8), 649-653.

Frenk, J., Chen, L., Bhutta, Z. A., Cohen, J., Crisp, N., Evans, T., .. \& Kistnasamy, B. (2010). Health professionals for a new century: transforming education to strengthen health systems in an interdependent world. The Lancet, 376(9756), 1923-1958.

Gontijo, E. D., Alvim, C. G., \& Castro Lima, M. E. C. de (2015). Manual de avaliação da aprendizagem no curso de graduação em Medicina. Revista Docência do Ensino Superior, 5(1), 205-325. 
Gontijo, E. D., Alvim, C., Megale, L., Melo, J. R. C., \& Lima, M. E. C. C. (2013). Matriz de competências essenciais para a formação e avaliação de desempenho de estudantes de medicina. Revista Brasileira de Educação Médica, 37(4), 526-539.

Jug, R., Jiang, X. S., \& Bean, S. M. (2019). Giving and receiving effective feedback: A review article and how-to guide. Archives of Pathology \& Laboratory Medicine, 143(2), 244-250.

Kaplan, L. M., Golden, A., Jinnett, K., Kolotkin, R. L., Kyle, T. K., Look, M.,... \& Stevenin, B. (2018). Perceptions of barriers to effective obesity care: results from the national ACTION study. Obesity, 26(1), 61-69.

Leedham-Green, K. E., Pound, R., \& Wylie, A. (2016). Enabling tomorrow's doctors to address obesity in a GP consultation: an action research project. Education for Primary Care, 27(6), 455-461.

Malatskey, L., Essa-Hadad, J., Willis, T. A., \& Rudolf, M. C. (2019). Leading healthy lives: lifestyle medicine for medical students. American Journal of Lifestyle Medicine, 13(2), 213-219.

Matharu, K., Shapiro, J. F., Hammer, R. R., Kravitz, R. L., Wilson, M. D., \& Fitzgerald, F. T. (2014). Reducing obesity prejudice in medical education. Education for Health: Change in Learning and Practice, 27(3), 231-237.

Medeiros, M. M. (2019). O manejo clínico do excesso de peso: saberes dos estudantes de medicina (Dissertação de Mestrado). Faculdade de Medicina, Universidade Federal de Alagoas, Maceió.

Mogre, V., Scherpbier, A. J., Stevens, F., Aryee, P., Cherry, M. G., \& Dornan, T. (2016). Realist synthesis of educational interventions to improve nutrition care competencies and delivery by doctors and other healthcare professionals. BMJ Open, 6(10), e010084.

Mogre, V., Stevens, F. C., Aryee, P. A., Amalba, A., \& Scherpbier, A. J. (2018). Why nutrition education is inadequate in the medical curriculum: a qualitative study of students' perspectives on barriers and strategies. BMC Medical Education, 18(1), 26.

Oberg, E. B., \& Frank, E. (2009). Physicians' health practices strongly influence patient health practices. The Journal of the Royal College of Physicians of Edinburgh, 39(4), 290-291.

Ockene, J. K., Ashe, K. M., Hayes, R. B., Churchill, L. C., Crawford, S. L., Geller, A. C.,... \& Ferguson, K. J. (2018). Design and rationale of the medical students learning weight management counseling skills (MSWeight) group randomized controlled trial. Contemporary Clinical Trials, 64, 58-66.

Pantenburg, B., Sikorski, C., Luppa, M., Schomerus, G., König, H. H., Werner, P. \& Riedel-Heller, S. G. (2012). Medical students' attitudes towards overweight and obesity. PloS One, 7(11), 1-7.

Reeves S., Perrier L., Goldman J., Freeth, D. \& Zwarenstein, M. (2013). Interprofessional education: effects on professional practice and health outcomes (update). Cochrane Database of Systematic Reviews, (3), CD002213.

Scolapio, J. S., DiBaise, J. K., Schwenk, W. F., Macke, M. E., \& Burdette, R. (2008). Advances and controversies in clinical nutrition: the education outcome of a live continuing medical education course. Nutrition in Clinical Practice, 23(1), 90-95.

Selltiz, C., Wrightsman, L., \& Cook, S. (1987). Métodos de Pesquisa nas Relações Sociais (Vol. 1, 2. ed.). São Paulo: E.P.U.

Vitolins, M. Z., Crandall, S., Miller, D., Ip, E., Marion, G., \& Spangler, J. G. (2012). Obesity educational interventions in US medical schools: a systematic review and identified gaps. Teaching and Learning in Medicine, 24(3), 267-272.

World Health Organization. (2010). Framework for action on interprofessional education and collaborative practice. Geneva: WHO.

Yanovski, S. Z., \& Yanovski, J. A. (2014). Long-term drug treatment for obesity: a systematic and clinical review. Jama, 311(1), 74-86.

Yin, R. K. (2016). Pesquisa qualitativa do início ao fim. Porto Alegre: Penso Editora. 